\title{
Neisseria mucosa
}

National Cancer Institute

\section{Source}

National Cancer Institute. Neisseria mucosa. NCI Thesaurus. Code C86606.

A species of aerobic, Gram-negative, diplococci shaped bacteria assigned to the phylum Proteobacteria. This species is catalase and oxidase positive, non-hemolytic, produces a yellowish pigment, reduces both nitrate and nitrite, synthesizes polysaccharides, and produces acid from glucose, maltose, fructose, and sucrose, but not mannose or lactose. N. mucosa is commensal in the human nasopharynx and is rarely pathogenic. 